\title{
Erratum: Half-Magnetic Topological Insulator with Magnetization-Induced Dirac Gap at a Selected Surface [Phys. Rev. X 11, 011039 (2021)]
}

Ruie Lu, Hongyi Sun, Shiv Kumar, Yuan Wang, Mingqiang Gu, Meng Zeng, Yu-Jie Hao, Jiayu Li, Jifeng Shao, Xiao-Ming Ma, Zhanyang Hao, Ke Zhang, Wumiti Mansuer, Jiawei Mei, Yue Zhao, Cai Liu, Ke Deng, Wen Huang, Bing Shen, Kenya Shimada, Eike F. Schwier, Chang Liu, Qihang Liu, and Chaoyu Chen®

(Q) (Received 8 May 2021; published 26 May 2021)

DOI: 10.1103/PhysRevX.11.029902

We recognize that the chiral surface edge state of a magnetic topological insulator with a gapless lateral surface state was first predicted by Chu et al. via tight-binding lattice models [1]. They also proposed an experimental scheme to measure the surface half-quantized Hall conductance. The DFT-calculated spectral functions in Fig. 4(d) are in good agreement with their work, providing substantial evidence to support the existence of the chiral surface edge states (i.e., the chiral hinge states in [1]) and axion insulator phase in this paper.

[1] R.-L. Chu, J. Shi, and S.-Q. Shen, Surface Edge State and Half-Quantized Hall Conductance in Topological Insulators, Phys. Rev. B 84, 085312 (2011).

Published by the American Physical Society under the terms of the Creative Commons Attribution 4.0 International license. Further distribution of this work must maintain attribution to the author(s) and the published articles title, journal citation, and DOI. 